\title{
Closed-Loop Insulin Delivery during Pregnancy in Women with Type 1 Diabetes
}

\author{
Zoe A. Stewart, M.D., Malgorzata E. Wilinska, Ph.D., Sara Hartnell, B.Sc., \\ Rosemary C. Temple, M.D., Gerry Rayman, M.D., Katharine P. Stanley, M.D., \\ David Simmons, M.D., Graham R. Law, Ph.D., Eleanor M. Scott, M.D., \\ Roman Hovorka, Ph.D., and Helen R. Murphy, M.D.
}

From the Wellcome Trust-Medical Research Council Institute of Metabolic Science, University of Cambridge (Z.A.S., M.E.W., R.H., H.R.M.), and Wolfson Diabetes and Endocrine Clinic, Cambridge University Hospitals NHS Foundation Trust (S.H., D.S., H.R.M.), Cambridge, the Elsie Bertram Diabetes Centre (R.C.T., H.R.M.) and the Department of Obstetrics and Gynaecology (K.P.S.), Norfolk and Norwich University Hospitals NHS Foundation Trust, and the Norwich Medical School, University of East Anglia (H.R.M.), Norwich, the Ipswich Diabetes Centre, Ipswich Hospital NHS Trust, Ipswich (G.R.), and the Division of Epidemiology and Biostatistics, Leeds Institute of Cardiovascular and Metabolic Medicine, University of Leeds, Leeds (G.R.L., E.M.S.) - all in the United Kingdom. Address reprint requests to Dr. Murphy at Norwich Medical School, University of East Anglia, Fl. 2, Bob Champion Research and Education Bldg., Norwich NR4 7UQ, United Kingdom, or at hm386@medschl .cam.ac.uk.

N EngIJ Med 2016;375:644-54. DOI: 10.1056/NEJMoa1602494

Copyright @ 2016 Massachusetts Medical Society.

\section{A BSTRACT}

\section{BACKGROUND}

In patients with type 1 diabetes who are not pregnant, closed-loop (automated) insulin delivery can provide better glycemic control than sensor-augmented pump therapy, but data are lacking on the efficacy, safety, and feasibility of closed-loop therapy during pregnancy.

\section{METHODS}

We performed an open-label, randomized, crossover study comparing overnight closed-loop therapy with sensor-augmented pump therapy, followed by a continuation phase in which the closed-loop system was used day and night. Sixteen pregnant women with type 1 diabetes completed 4 weeks of closed-loop pump therapy (intervention) and sensor-augmented pump therapy (control) in random order. During the continuation phase, 14 of the participants used the closed-loop system day and night until delivery. The primary outcome was the percentage of time that overnight glucose levels were within the target range (63 to $140 \mathrm{mg}$ per deciliter [3.5 to $7.8 \mathrm{mmol}$ per liter]).

\section{RESULTS}

The percentage of time that overnight glucose levels were in the target range was higher during closed-loop therapy than during control therapy $(74.7 \%$ vs. $59.5 \%$; absolute difference, 15.2 percentage points; 95\% confidence interval, 6.1 to 24.2 ; $\mathrm{P}=0.002)$. The overnight mean glucose level was lower during closed-loop therapy than during control therapy (119 vs. $133 \mathrm{mg}$ per deciliter [6.6 vs. $7.4 \mathrm{mmol}$ per liter], $\mathrm{P}=0.009$ ). There were no significant differences between closed-loop and control therapy in the percentage of time in which glucose levels were below the target range $(1.3 \%$ and $1.9 \%$, respectively; $\mathrm{P}=0.28)$, in insulin doses, or in adverseevent rates. During the continuation phase (up to 14.6 additional weeks, including antenatal hospitalizations, labor, and delivery), glucose levels were in the target range $68.7 \%$ of the time; the mean glucose level was $126 \mathrm{mg}$ per deciliter $(7.0 \mathrm{mmol}$ per liter). No episodes of severe hypoglycemia requiring third-party assistance occurred during either phase.

\section{CONCLUSIONS}

Overnight closed-loop therapy resulted in better glucose control than sensor-augmented pump therapy in pregnant women with type 1 diabetes. Women receiving day-and-night closed-loop therapy maintained glycemic control during a high proportion of the time in a period that encompassed antenatal hospital admission, labor, and delivery. (Funded by the National Institute for Health Research and others; Current Controlled Trials number, ISRCTN71510001.) 
OMPLICATIONS OF TYPE 1 DIABETES mellitus during pregnancy include increased rates of congenital anomaly, stillbirth, neonatal death, preterm delivery, and macrosomia. ${ }^{1}$ Congenital anomalies are associated with poor glycemic control around the time of conception, whereas the other complications are associated with maternal hyperglycemia that persists during pregnancy. ${ }^{2-5}$

Pregnant women with type 1 diabetes face particular challenges in trying to maintain tight glycemic control. Insulin requirements typically increase by a factor of 2 to 3 during the second and third trimesters, with substantial day-to-day variability, making the need for dose adjustments and their required magnitude unpredictable., ${ }^{6,7}$ Even with regular glucose monitoring, intensive insulin therapy, and glycated hemoglobin levels below $7 \%$, pregnant women with type 1 diabetes have glucose levels that are above the target range approximately half the time. ${ }^{8,9}$ They also have increased rates of hypoglycemia, ${ }^{6,10-12}$ with glucose levels that are below the target range for up to 3.5 hours per day, ${ }^{8}$ so the benefit of avoiding hyperglycemia for the infant must be weighed against the risk of hypoglycemia for the mother.

Technological advances in glucose monitoring and insulin delivery, including continuous glucose monitoring, insulin pumps, and sensor-augmented pump therapy, may allow for safer improvements in glycemic control. ${ }^{13}$ Closed-loop systems use a computer algorithm (a set of mathematical instructions) to adjust insulin-pump delivery in response to glucose measurements obtained from real-time continuous glucose monitors. ${ }^{14}$ These systems have been shown to improve glycemic control without increasing the risk of hypoglycemia under experimental conditions, ${ }^{15-17}$ in trials of supervised outpatient treatment, ${ }^{18-22}$ and in studies of unsupervised, self-administered treatment among patients who were not pregnant. ${ }^{23-25}$ Preliminary data suggest that closed-loop systems may maintain near-normal glucose levels and minimize the risk of nocturnal hypoglycemia among pregnant women with type 1 diabetes. ${ }^{26,27}$ We performed a 4-week, randomized, crossover study of an overnight closed-loop system, followed by a 14-week continuation phase of dayand-night closed-loop therapy, which encompassed pregnancy-related challenges, including antenatal hospital admission, labor, and delivery, as well as postnatal adaptation.

\section{METHODS}

\section{STUDY PARTICIPANTS}

We recruited pregnant women who had a history of type 1 diabetes mellitus for at least 12 months. Participants were 18 to 45 years of age, with a pregnancy between 8 and 24 weeks of gestation and a glycated hemoglobin level between 6.5 and $10.0 \%$. The women were receiving intensive insulin therapy administered by means of either multiple daily injections or an insulin pump. Women were excluded if they had conceived with the use of assisted reproductive technologies, were receiving concurrent treatment that might influence glucose control, had a multiple-gestation pregnancy, or had clinically significant nephropathy, neuropathy, or proliferative retinopathy, as judged by the investigator. Detailed inclusion and exclusion criteria are provided in the Supplementary Appendix, available with the full text of this article at NEJM.org. All participants provided written informed consent.

\section{STUDY OVERSIGHT}

The study protocol was approved by the East of England Research Ethics Committee of the Health Research Authority, with notification of no objection provided by the U.K. Medicines and Healthcare Products Regulatory Agency. The study was overseen by an independent data and safety monitoring board.

Abbott Diabetes Care provided discounted continuous-glucose-monitoring devices and consumables. Company representatives had no role in the design of the study; in the collection, handling, analysis, or interpretation of data; or in the decision to submit the manuscript for publication. The National Institute for Health Research and Abbott Diabetes Care received a copy of the manuscript before submission as a formality but did not provide input on the content.

\section{STUDY DESIGN}

The study was an open-label, multicenter, randomized, crossover trial. Participants were recruited from three U.K. National Health Service (NHS) sites.

After enrollment, participants were trained to use the study devices: a DANA Diabecare R Insulin Pump (SOOIL) and the FreeStyle Navigator II (Abbott Diabetes Care). After a run-in period of 2 to 4 weeks for device training and optimization 
of insulin doses, participants were randomly assigned in permuted blocks of 4 to either the overnight closed-loop system (intervention) or sensor-augmented pump therapy (control). Participants underwent a 2-week washout period after completing the first assigned intervention and before starting the second intervention. During the washout phase, participants used finger-stick testing, with or without continuous glucose monitoring or pump therapy, but could not use the closed-loop system.

After completion of the randomly assigned interventions, participants could choose to continue sensor-augmented pump therapy or the day-and-night closed-loop system with manually administered boluses before meals until delivery. This continuation phase provided a longer-term feasibility assessment of 24-hour closed-loop therapy while addressing the ethical questions that would be raised by withdrawal of an effective treatment during pregnancy.

Participants were advised to perform fingerstick glucose testing at least seven times daily, with standard glucose targets in both groups (63 to $99 \mathrm{mg}$ per deciliter [3.5 to $5.5 \mathrm{mmol}$ per liter] before a meal and $<140 \mathrm{mg}$ per deciliter [7.8 mmol per liter] 1 hour after a meal). Routine antenatal clinic visits were scheduled every 2 weeks, with fetal ultrasonographic assessments performed at 12, 20, 28, 32, and 36 weeks of gestation. There were no restrictions on physical activity, meals, or overseas travel, and no remote monitoring was performed. Participants had access to a 24-hour telephone line for assistance with technical difficulties.

C-peptide levels were measured when the serum glucose level was within the target range (63 to $140 \mathrm{mg}$ per deciliter [3.5 to $7.8 \mathrm{mmol}$ per liter]) at baseline, and glycated hemoglobin levels were measured at baseline, after each intervention phase, and at 28,32 , and 36 weeks of gestation. The study design is shown in Figure S1 in the Supplementary Appendix.

\section{CLOSED-LOOP SYSTEM}

During closed-loop therapy, a computer program, housed on a tablet computer, used continuous glucose measurements to determine an appropriate insulin dose. The insulin was delivered by means of an insulin pump every 12 minutes (Fig. S3 in the Supplementary Appendix). Pre- meal boluses were administered manually (15 to 30 minutes before the meal) as clinically indicated. ${ }^{28}$ To initialize closed-loop therapy, the participant's weight and total daily insulin dose were entered in the computer program. During the 4-week randomized phase, participants started closed-loop therapy after their evening meal and stopped before breakfast. During the dayand-night continuation phase, closed-loop therapy was used continuously, with manually administered boluses before meals. The device had to be within approximately $30 \mathrm{~m}$ of the participant in order to maintain connectivity. There were no programming changes in anticipation of antenatal glucocorticoid use, labor, or delivery.

\section{STUDY END POINTS}

The primary efficacy end point was the percentage of time that glucose was in the target range of 63 to $140 \mathrm{mg}$ per deciliter overnight, as recorded by means of continuous glucose monitoring during each 4-week study phase. Secondary efficacy end points were the mean glucose level, the percentages of time overnight and during the day and evening that glucose levels were above and below relevant thresholds, glucose variability, insulin dose, and glycated hemoglobin level.

Safety end points included the number and duration of hypoglycemic episodes (moderate or severe). Moderate hypoglycemia was defined as a glucose level of less than $63 \mathrm{mg}$ per deciliter for 20 minutes or longer, as measured by continuous glucose monitoring. A severe hypoglycemic episode was defined as an episode requiring third-party assistance.

The feasibility of day-and-night closed-loop therapy in the continuation phase (from the end of the crossover phase until delivery) was assessed on the basis of glucose measurements during sequential 4-week intervals and over the period as a whole. The same glucose targets and study end points were used during the crossover and continuation study phases.

\section{STATISTICAL ANALYSIS}

In our previous study of a closed-loop system with the use of sensor-augmented pump therapy in pregnant women with type 1 diabetes (median glycated hemoglobin level, $6.4 \%$ ), the mean ( \pm SD) percentage of time that glucose levels were in 
the target range was $61.7 \pm 24.9 \% .{ }^{27}$ We calculated that we would need to enroll 16 women for the current study to have a power of $80 \%$ to detect a $30 \%$ relative increase in the percentage of time that glucose levels were in the target range (from $62 \%$ with sensor-augmented pump therapy to $80 \%$ with the closed-loop system), at an alpha level of 0.05 (two-tailed). The standard deviation for the primary outcome was assumed to be $25 \% .{ }^{26,27}$

Statistical analyses were performed on an intention-to-treat basis, with data analyzed according to the study phase to which the participant had been assigned, regardless of adherence to the assigned intervention. We used linear mixed-effects models to estimate the percentage of overnight time that glucose levels were in the target range (response variable). The fixed effect of interest was whether there was a difference between sensor-augmented pump therapy and closed-loop therapy. Since the response variable was a repeated measure, we included nested random effects for the average time-in-target value for each study participant and for each 4-week time period for each participant. The fit of the model was not improved by including a term for either study phase-by-intervention interaction or autocorrelation of the response variable over time, and the estimated difference between study phases was not materially altered. Functional analysis of the continuous glucose data $^{29}$ was performed and adjusted for weeks of gestation and period effect. Sequential glucose measurements were modeled as trajectories by calculating continuous mathematical functions of glucose measurements. These trajectories were modeled by fitting $\mathrm{B}$-splines to the repeated measures. ${ }^{30} \mathrm{~A}$ two-sided significance level of 0.05 was used for both primary and secondary outcomes, without adjustment for multiple comparisons.

\section{RESULTS}

\section{STUDY PARTICIPANTS}

A total of 20 participants were recruited to participate in the study. Of these participants, 3 withdrew during the run-in training phase and 17 underwent randomization. One participant withdrew during her first study phase (sensoraugmented pump therapy) because of termination of pregnancy for trisomy 13 (a chromosomal

\begin{tabular}{|c|c|}
\hline Characteristic & Value \\
\hline Age $-y r$ & $34.1 \pm 4.6$ \\
\hline Body-mass index广 & $29.7 \pm 5.7$ \\
\hline Glycated hemoglobin — \% & $6.8 \pm 0.6$ \\
\hline Duration of diabetes $-\mathrm{yr}$ & $23.6 \pm 7.2$ \\
\hline Previous pump use - no. (\%) & $10(63)$ \\
\hline Previous use of continuous glucose monitoring - no. (\%) & $2(13)$ \\
\hline Total daily insulin dose $-U$ & $52.8 \pm 18.1$ \\
\hline \multicolumn{2}{|l|}{ Euglycemic C-peptide — pmol/liter } \\
\hline Median & 20 \\
\hline Interquartile range & $10-37$ \\
\hline Microalbuminuria - no. $(\%) \ddagger$ & $1(6)$ \\
\hline Weeks of gestation $\mathbb{S}$ & $14 \pm 3.3$ \\
\hline First pregnancy — no. (\%) 9 & $7(44)$ \\
\hline \multicolumn{2}{|l|}{ U.K. recruitment site - no. (\%) } \\
\hline Cambridge & $10(63)$ \\
\hline Norwich & $5(31)$ \\
\hline Ipswich & $1(6)$ \\
\hline
\end{tabular}

* Plus-minus values are means \pm SD.

$\uparrow$ The body-mass index is the weight in kilograms divided by the square of the height in meters.

$\uparrow$ Microalbuminuria at baseline was defined as an albumin:creatinine ratio that was higher than $3 \mathrm{mg}$ per millimole at the time that the pregnancy was confirmed.

\Data are shown for weeks of gestation at randomization. Participants were randomly assigned to one of the two intervention phases after 2 to 4 weeks of training, when insulin regimens were optimized and participants were confident in the use of the study insulin pump and continuous glucose monitor.

9 Among the nine women with previous pregnancies, five had previous pregnancy losses (five miscarriages and two stillbirths); one woman had a secondtrimester termination of pregnancy because of major fetal malformation, and two women had each had two preterm deliveries (before 34 weeks of gestation).

anomaly unrelated to diabetes). Sixteen participants completed both study phases and were included in the analyses (Table 1). Six participants were receiving multiple daily insulin injections, and 14 participants had no experience with continuous glucose monitoring before the study.

\section{STUDY OUTCOMES}

The percentage of overnight time that glucose values were within the target range was significantly higher with closed-loop therapy than with sensor-augmented pump therapy $(74.7 \%$ vs. $59.5 \%$; absolute difference, 15.2 percentage points; $95 \%$ confidence interval, 6.1 to $24.2 ; \mathrm{P}=0.002$ ) (Table 2 


\begin{tabular}{|c|c|c|c|c|}
\hline Variable & $\begin{array}{l}\text { Sensor-Augmented } \\
\text { Pump Therapy }\end{array}$ & $\begin{array}{l}\text { Closed-Loop } \\
\text { Insulin Delivery }\end{array}$ & $\begin{array}{l}\text { Absolute Difference } \\
\text { (95\% Cl) }\end{array}$ & P Value \\
\hline Glucose in target range ( $\%$ of time $) \dagger$ & 59.5 & 74.7 & $15.2(6.1$ to 24.2$)$ & 0.002 \\
\hline \multicolumn{5}{|l|}{ Glucose above target range (\% of time) } \\
\hline$>140 \mathrm{mg} / \mathrm{dl}$ & 38.6 & 24.0 & $-14.5(-24.2$ to -4.9$)$ & 0.005 \\
\hline$>180 \mathrm{mg} / \mathrm{dl}$ & 15.7 & 7.4 & $-8.3(-13.7$ to -3.0$)$ & 0.004 \\
\hline \multicolumn{5}{|l|}{ Glucose below target range ( $\%$ of time) } \\
\hline$<63 \mathrm{mg} / \mathrm{dl}$ & 1.9 & 1.3 & $-0.6(-1.7$ to 0.6$)$ & 0.28 \\
\hline$<50 \mathrm{mg} / \mathrm{dl}$ & 0.6 & 0.3 & $-0.2(-0.9$ to -0.4$)$ & 0.45 \\
\hline $\begin{array}{l}\text { Median no. of hypoglycemic episodes } \\
\text { (range) } t\end{array}$ & $2.5(0$ to 15.0$)$ & $3.0(0$ to 6.0$)$ & & 0.68 \\
\hline Mean low blood glucose index $\mathbb{}$ & 1.3 & 1.3 & $0.1(-0.4$ to 0.5$)$ & 0.78 \\
\hline Mean glucose (mg/dl) & 133 & 119 & $-14(-23$ to -4$)$ & 0.009 \\
\hline \multicolumn{5}{|l|}{$\begin{array}{l}\text { Median area under the curve (interquar- } \\
\text { tile range) }\end{array}$} \\
\hline Glucose $>140 \mathrm{mg} / \mathrm{dl}$ & 147.7 (40.2 to 322.4 ) & 39.2 (9.9 to 142.2 ) & & 0.07 \\
\hline Glucose $>121 \mathrm{mg} / \mathrm{dl}$ & 383.8 (222.0 to 608.9$)$ & 169.6 (98.5 to 413.7$)$ & & 0.04 \\
\hline Glucose $<63 \mathrm{mg} / \mathrm{dl}$ & 0 & 0 & & \\
\hline Glucose $<50 \mathrm{mg} / \mathrm{dl}$ & 0 & 0 & & \\
\hline $\begin{array}{l}\text { Standard deviation for sensor-recorded } \\
\text { glucose }(\mathrm{mg} / \mathrm{dl})\end{array}$ & 27 & 25 & $-2(-4$ to 0$)$ & 0.13 \\
\hline \multicolumn{5}{|c|}{$\begin{array}{l}\text { * The overnight period in the crossover phase of the study was from } 11 \text { p.m. to } 7 \text { a.m. To convert values for glucose to millimoles per liter, } \\
\text { multiply by } 0.05551 \text {. } \\
\text { The target range for glucose was } 63 \text { to } 140 \mathrm{mg} \text { per deciliter. The primary efficacy end point was the percentage of time that glucose was in } \\
\text { the target range overnight, as recorded by continuous glucose monitoring during each 4-week study phase. The reported values were de- } \\
\text { rived from linear mixed-effects models. } \\
\text { A hypoglycemic episode was defined as a blood glucose level below } 63 \mathrm{mg} \text { per deciliter for a period of } 20 \text { minutes or longer. } \\
\text { I The low blood glucose index assesses the duration and extent of hypoglycemia. Vvalues of less than } 2.5 \text { indicate a low risk of severe hypo- } \\
\text { glycemia, values of } 2.5 \text { to } 5 \text { indicate a moderate risk, and values of more than } 5 \text { indicate a high risk. The reported values were derived from } \\
\text { linear mixed-effects models. }\end{array}$} \\
\hline
\end{tabular}

and Fig. 1). The mean glucose level was significantly lower during closed-loop therapy than during sensor-augmented pump therapy, both overnight (119 vs. $133 \mathrm{mg}$ per deciliter [6.6 vs. $7.4 \mathrm{mmol}$ per liter], $\mathrm{P}=0.009$ ) (Table 2) and over a 24-hour period (128 vs. $137 \mathrm{mg}$ per deciliter [7.1 vs. 7.6 mmol per liter], $\mathrm{P}<0.001$ ) (Tables 2 and 3).

The incidence of maternal hyperglycemia was lower during closed-loop therapy than during sensor-augmented pump therapy, both overnight and over a 24-hour period. The incidence of substantial nocturnal hyperglycemia (glucose level, $>180 \mathrm{mg}$ per deciliter [10.0 mmol per liter]) was significantly lower during overnight closed-loop therapy than during sensor-augmented pump therapy (Table 2). Functional data analysis showed that overnight closed-loop therapy was associat- ed with a significantly lower glucose level for a total time of 7 hours and 20 minutes (between 1:50 a.m. and 9:10 a.m.), with no significant effect for gestational age or study phase (Fig. S4 in the Supplementary Appendix).

The percentage of time that glucose levels were in the hypoglycemic range $(<63 \mathrm{mg}$ per deciliter) was low $(<2 \%)$, with no significant differences between the two phases of the crossover study. There were no episodes of severe hypoglycemia during either phase.

Glycated hemoglobin levels declined from baseline to the end of both phases, with no significant difference in changes between the two phases of the crossover study $(\mathrm{P}=0.67)$. Total daily insulin doses were similar in the two phases, although insulin delivery was significantly more variable 


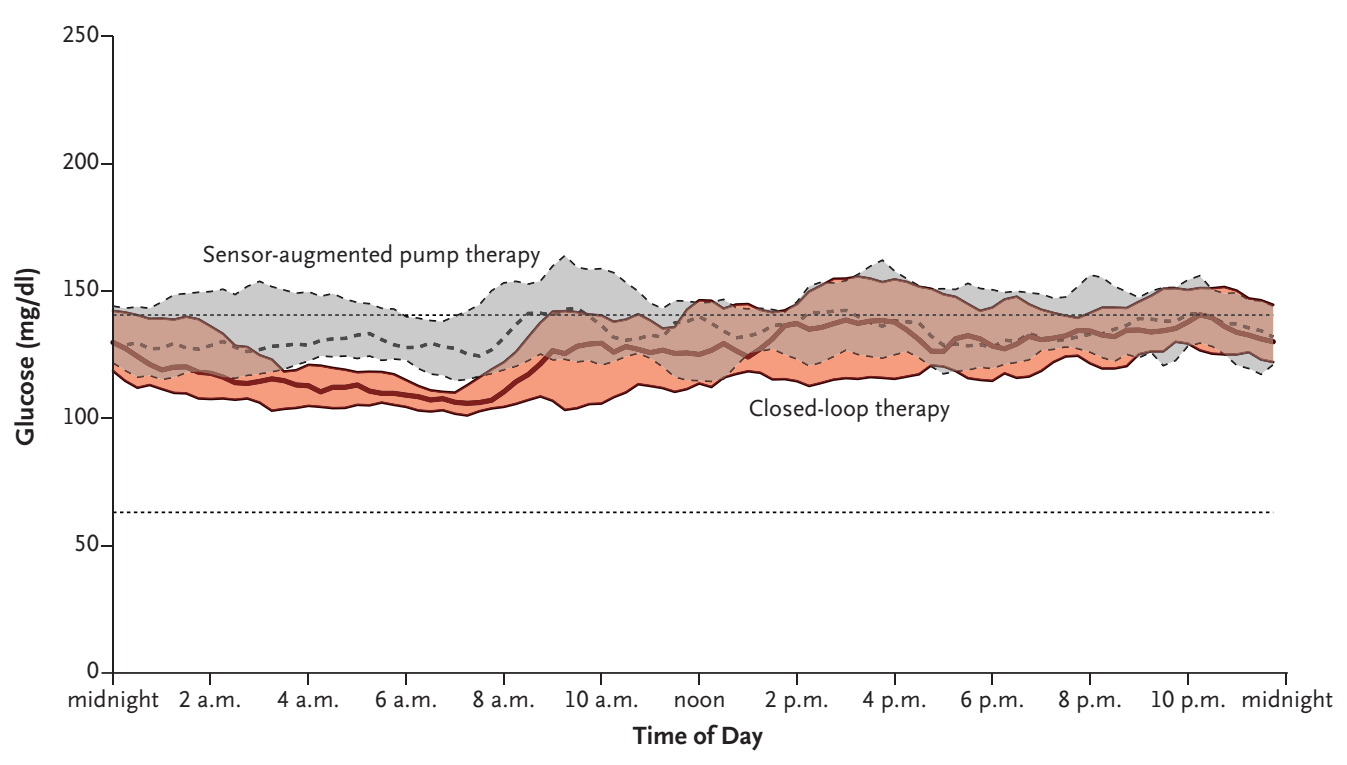

Figure 1. Median Sensor-Recorded Glucose Values over a 24-Hour Period with Sensor-Augmented Pump Therapy and Closed-Loop Insulin Delivery.

The target range for glucose values during pregnancy is 63 to $140 \mathrm{mg}$ per deciliter, indicated by the two horizontal dotted lines. Functional data analysis confirmed significant differences in glucose control between the two intervention phases for a total time of 7 hours 20 minutes (from 1:50 a.m to 9:10 a.m.), with no effect of gestation and no study-phase interaction. Shading indicates the interquartile range. To convert values for glucose to millimoles per liter, multiply by 0.05551 .

Table 3. Comparison of Sensor-Augmented Pump Therapy and Closed-Loop Insulin Delivery during the Day and Evening in the Crossover Phase of the Study.*

Variable

Glucose in target range (\% of time)

Glucose above target range (\% of time)

$>140 \mathrm{mg} / \mathrm{dl}$
$>180 \mathrm{mg} / \mathrm{dl}$

Glucose below target range (\% of time)

$$
\begin{aligned}
& <63 \mathrm{mg} / \mathrm{dl} \\
& <50 \mathrm{mg} / \mathrm{dl}
\end{aligned}
$$

Median no. of hypoglycemic episodes (range)

Mean glucose $(\mathrm{mg} / \mathrm{dl})$

Total insulin dose ( $\mathrm{U} /$ day)

Sensor wear (hr)
Sensor-Augmented Pump Therapy 56.8 40.9

$$
1.8
$$

12.0 (2.0 to 26.0 )

137

20.6

\section{Closed-Loop Insulin Delivery}

66.3

31.6

12.6

1.9

0.4

11.0 (0 to 37.0$)$

128

59.8

21.1

\section{Absolute Difference}

(95\% Cl)

9.4 (5.1 to 13.8 )

$-9.4(-13.7$ to -5.0$)$

$-4.7(-7.3$ to -2.1$)$

$<0.001$

0.001

* The closed-loop system was active overnight only during the crossover phase of the study, and premeal boluses were given manually (15 to 30 minutes before a meal). The reported values were derived from linear mixed-effects models.

during closed-loop therapy than during sensoraugmented pump therapy $(\mathrm{P}<0.001)$ (Table S1 in the Supplementary Appendix). The results were similar among experienced pump users and those with no previous experience in pump use (Table S2 in the Supplementary Appendix). 


\section{ADVERSE EVENTS}

There were 26 adverse events (14 skin reactions and 12 minor illnesses), with no significant differences between closed-loop and sensoraugmented pump therapy. There were 95 device deficiencies (18 during sensor-augmented pump therapy, 21 during closed-loop therapy, and 56 during the run-in and continuation phases), none of which resulted in severe hypoglycemia or hyperglycemia (Table S3 in the Supplementary Appendix). There were 8 serious adverse events. One of these events, which occurred during the randomized phase of closedloop therapy, was a hospital admission after an episode of self-treated hypoglycemia resulting from recurrent vomiting; however, this event occurred during the daytime, when the closedloop system was not operational. Another serious adverse event (vomiting due to gastroenteritis) occurred during the run-in training phase, and 6 events occurred during the continuation phase (Table S4 in the Supplementary Appendix). No serious adverse events were considered by the study investigators to be devicerelated.

\section{FEASIBILITY OF DAY-AND-NIGHT CLOSED-LOOP THERAPY THROUGHOUT PREGNANCY}

Fourteen participants chose to continue using the closed-loop system after they finished the crossover studies, which provided up to an additional 14.6 weeks (median, 11.6 weeks [interquartile range, 7.1 to 12.7]) of day-and-night closedloop use for feasibility assessment. Throughout the continuation phase with the closed-loop system, glucose levels were in the target range a mean of $68.7 \%$ of the time, and the mean glucose level was $126 \mathrm{mg}$ per deciliter $(7.0 \mathrm{mmol}$ per liter).

The median glucose levels were $124,128,124$, and $115 \mathrm{mg}$ per deciliter $(6.9,7.1,6.9$, and $6.4 \mathrm{mmol}$ per liter) at gestational ages of 24 weeks to 27 weeks 6 days, 28 weeks to 31 weeks 6 days, 32 weeks to 35 weeks 6 days, and 36 weeks or more, respectively (Tables S5 and S6 in the Supplementary Appendix). The median percentages of time that glucose levels were in the target range were $70.9 \%, 67.6 \%, 67.8 \%$, and $77.3 \%$ at the respective gestational ages. The median percentages of time that glucose levels were below the target ranged from 1.2 to $2.1 \%$ throughout pregnancy.

\section{CLOSED-LOOP THERAPY DURING LABOR} AND DELIVERY

Fourteen women continued closed-loop therapy during labor and delivery (Fig. 2). In the 24 hours before delivery, these women had a median glucose level of $110 \mathrm{mg}$ per deciliter (interquartile range, 104 to 128 [6.1 mmol per liter; interquartile range, 5.8 to 7.1]), with glucose levels in the target range of 63 to $140 \mathrm{mg}$ per deciliter $86.8 \%$ of the time (interquartile range, 59.6 to 94.1 ) and glucose levels below the target range $0.5 \%$ of the time (interquartile range, 0 to 1.8). In the first 48 hours after delivery, these women had a median glucose level of $117 \mathrm{mg}$ per deciliter (interquartile range, 104 to 137 [6.5 $\mathrm{mmol}$ per liter; interquartile range, 5.8 to 5.6$]$ ), with glucose levels in the target range $73.7 \%$ of the time (interquartile range, 61.4 to 86.0 ) and values below the target range $0 \%$ of the time (interquartile range, 0 to 0.5 ). The median total daily insulin dose was $53.6 \%$ of the predelivery dose (interquartile range, 48.6 to 73.6 ), with substantial variation among the participants (Table S7 in the Supplementary Appendix). There were no episodes of maternal hypoglycemia during the 24 hours before or 48 hours after delivery.

\section{OBSTETRICAL AND NEONATAL OUTCOMES}

The median gestational age at delivery was 36.9 weeks (interquartile range, 34.5 to 37.7). Preeclampsia developed in 5 participants, including 1 with the HELLP syndrome (hemolysis, elevated liver enzyme levels, and a low platelet count). Cesarean section was performed in 15 partici-

Figure 2 (facing page). Glycemic Control during Labor and Delivery in the 14 Participants Who Continued to Use Closed-Loop Insulin Delivery.

Data are shown for the 24-hour period before delivery and the 48-hour period after delivery, with delivery at time 0 . Participants 5, 6, 8, 9, and 15 received antenatal glucocorticoids for fetal lung maturation. Participant 3 had a urinary tract infection in the antepartum period, which was the reason for her delivery. Participant 15 had Addison's disease and was treated with high-dose glucocorticoids in the immediate postpartum period. Carbohydrate-to-insulin ratios were changed as soon as possible after delivery, and prandial insulin was withheld for the first meal after delivery. The dark green shading indicates the target glycemic range of 63 to $140 \mathrm{mg}$ per deciliter ( 3.5 to $7.8 \mathrm{mmol}$ per liter). The light green shading indicates an elevated glycemic range of 141 to $180 \mathrm{mg}$ per deciliter ( 7.8 to $10.0 \mathrm{mmol}$ per liter). 


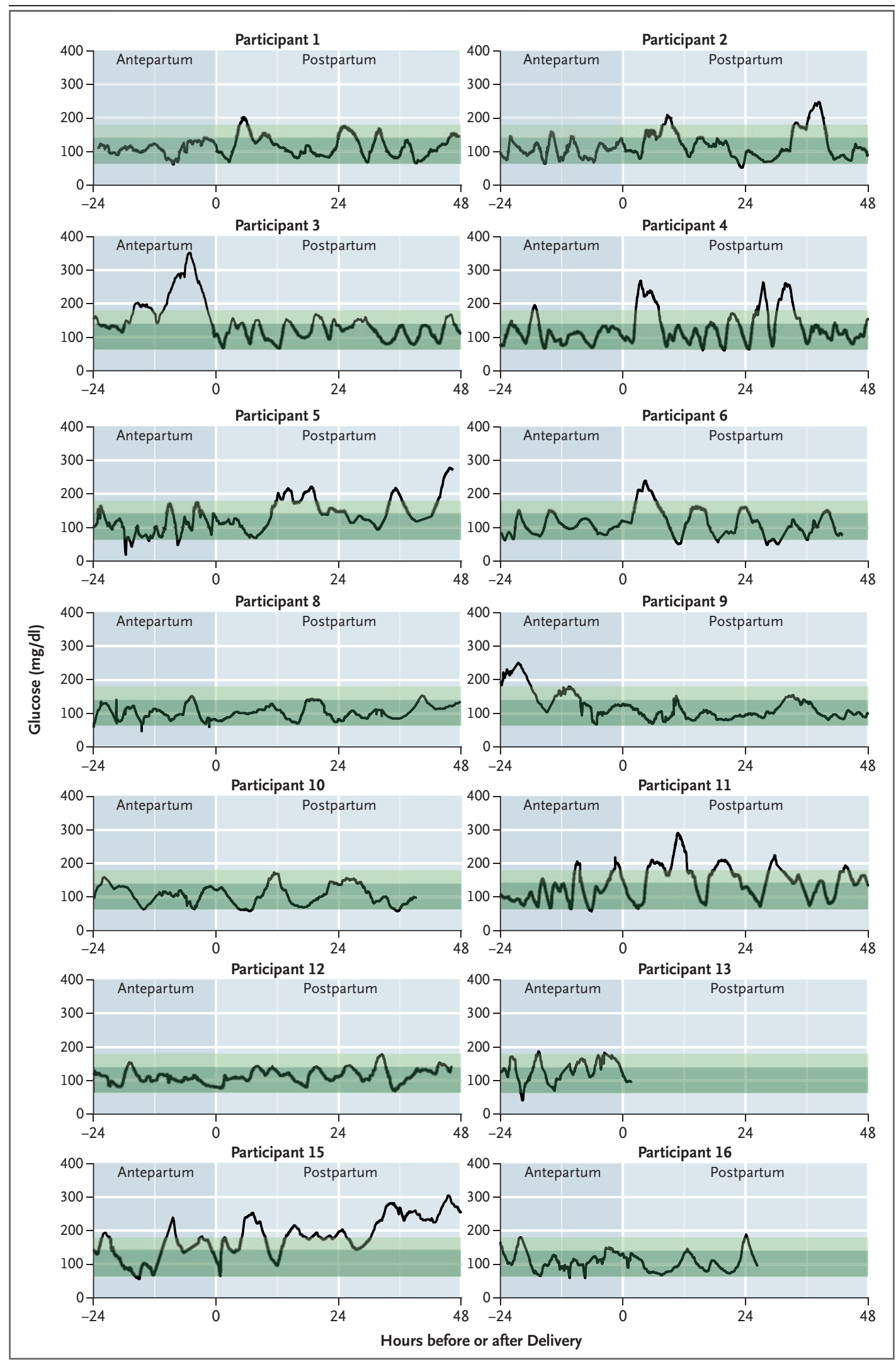


pants ( 2 of whom were under general anesthesia); 10 of the procedures were performed before the onset of labor. One participant underwent an ovarian cystectomy during cesarean section. Seven participants delivered before 37 weeks of gestation (with 4 of the 7 participants delivering before 34 weeks of gestation), and in 6 participants, antenatal glucocorticoids were administered for fetal lung maturation. On days 1, 2, and 3 after glucocorticoid administration, glucose levels were in the target range a median of $58.1 \%, 59.1 \%$, and $70.9 \%$ of the time, respectively. The closed-loop system delivered a median of 169 to $178 \%$ of the pre-glucocorticoid insulin dose, although there was substantial variation among the participants (Tables S8 and S10 in the Supplementary Appendix).

The median birth weight was $3588 \mathrm{~g}$ (interquartile range, 2670 to 3998). Thirteen of the 16 infants had a birth weight (corrected for sex and gestational age) that was above the 90th percentile, according to population-based U.K. data. ${ }^{31}$ Twelve infants received neonatal intensive care, 11 of whom were treated with intravenous dextrose for neonatal hypoglycemia. Details regarding the obstetrical outcomes are provided in Table S11 in the Supplementary Appendix.

DISCUSSION

As compared with sensor-augmented pump therapy, overnight closed-loop therapy resulted in a significant increase (by 15 percentage points) in the percentage of time that glucose levels were in the target range for pregnancy over a 24-hour period, as well as in a lower mean glucose level. These improvements were achieved without an increased incidence of hypoglycemia or an increase in the total insulin dose but with more variable insulin delivery to minimize hyperglycemic excursions.

After the crossover phase of the study, 14 participants continued to use day-and-night closedloop therapy for up to an additional 14.6 weeks, with the results showing the feasibility of 24-hour use of the closed-loop system during pregnancy, delivery, and in the first 48 hours after delivery. These observations are important because pregnancy provides challenges to the use of a closedloop system, some of which are an integral part of pregnancy (e.g., week-by-week changes in insulin resistance and pharmacokinetics, ${ }^{7}$ labor and delivery, and the rapid decrease in insulin requirements after delivery). Further challenges arise from antenatal admissions, administration of glucocorticoids for fetal lung maturation, and the use of anesthesia for cesarean section. Previous outpatient studies of closed-loop therapy ${ }^{18,23}$ have focused on relatively steady-state diabetes in the absence of pregnancy. The closed-loop system maintained maternal glycemic control in study participants throughout pregnancy and delivery and associated challenges without any changes in programming to the system and without any episodes of severe hypoglycemia requiring third-party assistance.

The randomized crossover design of our study minimized the effect of confounding factors. There was no significant effect of study phase or gestational age, suggesting that the closed-loop system adjusted insulin consistently. We included 14 participants who had no previous experience with sensor-augmented pumps, 6 of whom had never used an insulin pump of any kind. The glycemic-control outcomes for these participants were similar to the outcomes for the participants who were experienced pump users.

Our findings build on recent trials showing that a closed-loop system, as compared with sensor-augmented pump therapy, improved glycemic control, without increases in hypoglycemic episodes or the insulin dose. ${ }^{18,23}$ The glucose control achieved during our control phase was similar to that achieved with closed-loop interventions among patients who were not pregnant. This observation probably reflects the strong motivation to maintain glucose control during pregnancy and tighter glycemic targets. Despite impressive glycemic control with sensor-augmented pump therapy, closed-loop therapy still generated substantial improvements when used overnight.

Our sample was small but included women with a long duration of diabetes and substantial prior obstetrical morbidity, including five spontaneous pregnancy losses, one second-trimester termination, four early preterm deliveries, and two stillbirths. Such factors perhaps contributed to the high rate of preeclampsia. Among the infants, there were high incidences of birth weight above the 90th percentile for gestational age and neonatal hypoglycemia, despite the good glycemic control achieved. Fetal hyperinsulinemia and increased placental fuel transfer can persist 
with apparently normal maternal glycemia and may in part explain these outcomes. ${ }^{32}$ Larger trials of closed-loop therapy for a longer period are needed to evaluate the effects of this therapy on obstetrical and neonatal outcomes.

In conclusion, our crossover trial showed that overnight closed-loop therapy, as compared with sensor-augmented pump therapy, resulted in improved glucose control during pregnancy in women with type 1 diabetes. In the continuation phase, women receiving day-and-night closed-loop therapy maintained glycemic control during a high percentage of the time in a period that encompassed antenatal hospital admission, labor, and delivery.

The views expressed in this article are those of the authors and not necessarily those of the NHS, the National Institute for Health Research, or the U.K. Department of Health.
Supported by a grant from the National Institute for Health Research (NIHR) (CDF 2013-06-035, to Dr. Murphy); grants from Diabetes UK (BDA 07/0003551), Gates Cambridge Trust, and Jean Hailes for Women's Health; and a grant from the NIHR Cambridge Biomedical Research Centre and a Wellcome Strategic Award (100574/Z/12/Z, to Dr. Hovorka).

Disclosure forms provided by the authors are available with the full text of this article at NEJM.org.

We thank the study participants; key diabetes and obstetrical clinical staff at Cambridge University Hospital NHS Foundation Trust (Charlotte Patient, Caroline Byrne, Katy Davenport, Shazia Bhatti, Kerry Stubbington, and Ruth Harding), Norfolk and Norwich University Hospital (Katherine Duffield, Jeremy Turner, Alastair McKelvey, Sue Land, and Leanne Piper), Ipswich Hospital NHS Trust (Damian Morris, Duncan Fowler, Susan Mitchell, Josephine Rosier, and Balroop Johal), and Derby Teaching Hospitals NHS Foundation Trust (Paru King and Emma Wilmot); the wider artificial pancreas team for technical and device support (Martin Tauschmann, Janet Allen, and Hood Thabit), for equipment procurement (Sam Goode), and for administrative support and submissions to the Medicine and Healthcare Products Regulatory Agency (Jenni Curtis and Josephine Hayes); and the data safety and monitoring board for study oversight (Peter Hammond [chair], Michael Maresh, and Anne Dornhorst).

\section{REFERENCES}

1. Casson IF, Clarke CA, Howard CV, et al. Outcomes of pregnancy in insulin dependent diabetic women: results of a five year population cohort study. BMJ 1997; 315:275-8.

2. Kitzmiller JL, Gavin LA, Gin GD, Jovanovic-Peterson L, Main EK, Zigrang WD. Preconception care of diabetes: glycemic control prevents congenital anomalies. JAMA 1991;265:731-6.

3. Confidential Enquiry into Maternal and Child Health (CEMACH). Diabetes in pregnancy: are we providing the best care? Findings of a national enquiry: England, Wales and Northern Ireland. London: CEMACH, February 2007.

4. Middleton P, Crowther CA, Simmonds L. Different intensities of glycaemic control for pregnant women with pre-existing diabetes. Cochrane Database Syst Rev 2012;8:CD008540.

5. National Pregnancy in Diabetes (NIPD) audit. West Yorkshire, United Kingdom: Health and Social Care Information Centre, 2015 (http://www.hscic.gov.uk/npid). 6. García-Patterson A, Gich I, Amini SB, Catalano PM, de Leiva A, Corcoy R. Insulin requirements throughout pregnancy in women with type 1 diabetes mellitus: three changes of direction. Diabetologia 2010;53:446-51.

7. Goudie RJ, Lunn D, Hovorka R, Murphy HR. Pharmacokinetics of insulin as part in pregnant women with type 1 diabetes: every day is different. Diabetes Care 2014;37(6):e121-2.

8. Murphy HR, Rayman G, Duffield K, et al. Changes in the glycemic profiles of women with type 1 and type 2 diabetes during pregnancy. Diabetes Care 2007;30: 2785-91.

9. Kerssen A, Evers IM, de Valk HW,
Visser GH. Poor glucose control in women with type 1 diabetes mellitus and 'safe' hemoglobin A1c values in the first trimester of pregnancy. J Matern Fetal Neonatal Med 2003;13:309-13.

10. Ringholm L, Pedersen-Bjergaard U, Thorsteinsson B, Damm P, Mathiesen ER. Hypoglycaemia during pregnancy in women with Type 1 diabetes. Diabet Med 2012;29:558-66.

11. ter Braak EWMT, Evers IM, Willem Erkelens D, Visser GH. Maternal hypoglycemia during pregnancy in type 1 diabetes: maternal and fetal consequences. Diabetes Metab Res Rev 2002;18:96-105. 12. Evers IM, ter Braak EWMT, de Valk HW, van Der Schoot B, Janssen N, Visser GHA. Risk indicators predictive for severe hypoglycemia during the first trimester of type 1 diabetic pregnancy. Diabetes Care 2002;25:554-9.

13. Combs CA. Continuous glucose monitoring and insulin pump therapy for diabetes in pregnancy. J Matern Fetal Neonatal Med 2012;25:2025-7.

14. Kropff J, DeVries JH. Continuous glucose monitoring, future products, and update on worldwide artificial pancreas projects. Diabetes Technol Ther 2016;18: Suppl 2:S253-63.

15. Breton M, Farret A, Bruttomesso D, et al. Fully integrated artificial pancreas in type 1 diabetes: modular closed-loop glucose control maintains near normoglycemia. Diabetes 2012;61:2230-7.

16. Hovorka R, Kumareswaran K, Harris J, et al. Overnight closed loop insulin delivery (artificial pancreas) in adults with type 1 diabetes: crossover randomised controlled studies. BMJ 2011;342:d1855. 17. Hovorka R, Allen JM, Elleri D, et al. Manual closed-loop insulin delivery in children and adolescents with type 1 diabetes: a phase 2 randomised crossover trial. Lancet 2010;375:743-51.

18. Russell SJ, El-Khatib FH, Sinha M, et al. Outpatient glycemic control with a bionic pancreas in type 1 diabetes. $\mathrm{N}$ Engl J Med 2014;371:313-25.

19. Kovatchev BP, Renard E, Cobelli C, et al. Feasibility of outpatient fully integrated closed-loop control: first studies of wearable artificial pancreas. Diabetes Care 2013;36:1851-8.

20. Dassau E, Zisser H, Harvey RA, et al. Clinical evaluation of a personalized artificial pancreas. Diabetes Care 2013;36: 801-9.

21. Nimri R, Muller I, Atlas E, et al. MD-Logic overnight control for 6 weeks of home use in patients with type 1 diabetes: randomized crossover trial. Diabetes Care 2014;37:3025-32.

22. Ly TT, Roy A, Grosman B, et al. Day and night closed-loop control using the integrated Medtronic hybrid closed-loop system in type 1 diabetes at diabetes camp. Diabetes Care 2015;38:1205-11.

23. Thabit H, Tauschmann M, Allen JM, et al. Home use of an artificial beta cell in type 1 diabetes. N Engl J Med 2015;373: 2129-40.

24. Thabit H, Lubina-Solomon A, Stadler $\mathrm{M}$, et al. Home use of closed-loop insulin delivery for overnight glucose control in adults with type 1 diabetes: a 4-week, multicentre, randomised crossover study. Lancet Diabetes Endocrinol 2014;2:701-9. 25. Hovorka R, Elleri D, Thabit H, et al. Overnight closed-loop insulin delivery in young people with type 1 diabetes: a freeliving, randomized clinical trial. Diabetes Care 2014;37:1204-11.

26. Murphy HR, Elleri D, Allen JM, et al. 
Closed-loop insulin delivery during pregnancy complicated by type 1 diabetes. Diabetes Care 2011;34:406-11.

glycaemia in women with type 1 diabetes during pregnancy. Diabetologia 2012;55: 282-93.

27. Murphy HR, Kumareswaran K, Elleri D, 29. Law GR, Ellison GT, Secher AL, et al. et al. Safety and efficacy of 24-h closedloop insulin delivery in well-controlled pregnant women with type 1 diabetes: a randomized crossover case series. Diabetes Care 2011;34:2527-9.

28. Murphy HR, Elleri D, Allen JM, et al. Pathophysiology of postprandial hyperAnalysis of continuous glucose monitoring in pregnant women with diabetes: distinct temporal patterns of glucose associated with large-for-gestationalage infants. Diabetes Care 2015;38:1319 25.

30. Ramsay JO, Hooker G, Graves S. Func- tional data analysis with $\mathrm{R}$ and MATLAB. New York: Dordrecht Springer, 2009.

31. Freeman JV, Cole TJ, Chinn S, Jones PR, White EM, Preece MA. Cross sectional stature and weight reference curves for the UK, 1990. Arch Dis Child 1995;73:17-24 32. Desoye G, Nolan CJ. The fetal glucose steal: an underappreciated phenomenon in diabetic pregnancy. Diabetologia 2016; 59:1089-94.

Copyright (๑) 2016 Massachusetts Medical Society.

JOURNAL ARCHIVE AT NEJM.ORC

Every article published by the Journal is now available at NEJM.org, beginning with the first article published in January 1812. The entire archive is fully searchable, and browsing of titles and tables of contents is easy and available to all.

Individual subscribers are entitled to free 24-hour access to 50 archive articles per year. Access to content in the archive is available on a per-article basis and is also being provided through many institutional subscriptions. 